\title{
Review of: "Naked mole-rat brown fat thermogenesis is diminished during hypoxia through a rapid decrease in UCP1"
}

Jin-Song Liu ${ }^{1}$

1 Wenzhou University

Potential competing interests: The author(s) declared that no potential competing interests exist.

In this paper, the changes and causes of thermogenesis in brown adipose tissue of naked mole rat during hypoxia were analyzed. This is a well written, concise paper on gives an overview of adaptation of naked mole rat to hypoxia. Both the number and gene expression of mitochondrial UCP1 in BAT are dependent on the presence of $T_{3}$ and NE (Park et al., 1989; Lanni et al.,2003). Therefore, one thing I was hoping to read was whether there were corresponding changes in the secretion of these hormones in anoxic environment. 